\title{
Electron-Electron Correlation in Graphite: A Combined Angle-Resolved Photoemission and First-Principles Study
}

\author{
A. Grüneis, ${ }^{1}$ C. Attaccalite, ${ }^{2}$ T. Pichler, ${ }^{1}$ V. Zabolotnyy, ${ }^{1}$ H. Shiozawa, ${ }^{1}$ S. L. Molodtsov,${ }^{3}$ D. Inosov, ${ }^{1}$ A. Koitzsch, ${ }^{1}$ \\ M. Knupfer, ${ }^{1}$ J. Schiessling, ${ }^{4}$ R. Follath, ${ }^{5}$ R. Weber, ${ }^{5}$ P. Rudolf, ${ }^{6}$ L. Wirtz, ${ }^{2}$ and A. Rubio ${ }^{7}$ \\ ${ }^{1}$ IFW Dresden, P.O. Box 270116, D- 01171 Dresden, Germany \\ ${ }^{2}$ Institute for Electronics, Microelectronics, and Nanotechnology, B.P. 60069, 59652 Villeneuve d'Ascq Cedex, France \\ ${ }^{3}$ Institut für Festkörperphysik, TU Dresden, Mommsenstrasse 13, D-01069 Dresden, Germany \\ ${ }^{4}$ Department of Physics, Uppsala University, P.O. Box 53075 121, Uppsala, Sweden \\ ${ }^{5}$ BESSY II, Albert-Einstein-Strasse 15, 12489 Berlin, Germany \\ ${ }^{6}$ Zernike Institute for Advanced Materials, Rijksuniversiteit Groningen, Nijenborgh 4, NL-9747 AG, Groningen, The Netherlands \\ ${ }^{7}$ European Theoretical Spectroscopy Facility and Department of Material Physics, University of the Basque Country, \\ 20018 San Sebastian, Spain
}

(Received 18 April 2007; published 22 January 2008)

\begin{abstract}
The full three-dimensional dispersion of the $\pi$ bands, Fermi velocities, and effective masses are measured with angle-resolved photoemission spectroscopy and compared to first-principles calculations. The band structure by density-functional theory underestimates the slope of the bands and the trigonal warping effect. Including electron-electron correlation on the level of the $G W$ approximation, however, yields remarkable improvement in the vicinity of the Fermi level. This demonstrates the breakdown of the independent electron picture in semimetallic graphite and points toward a pronounced role of electron correlation for the interpretation of transport experiments and double-resonant Raman scattering for a wide range of carbon based materials.
\end{abstract}

DOI: 10.1103/PhysRevLett.100.037601

Recently graphene has been investigated as a prototype system to address basic questions of quantum mechanics [1-3] (relativistic Dirac fermions) as well as for high speed semimetal field effect transistors in emerging nanoelectronic devices [4]. Many of these results are based on its peculiar electronic properties, i.e., an isotropic and linear dispersion close to the Fermi level $\left(E_{F}\right)$. In low dimensional and strongly anisotropic systems correlation effects play a crucial role in understanding and describing the electronic band structure. Kinks in the quasiparticle (QP) dispersions and lifetimes were observed and interpreted as band renormalization due to electron-phonon [5] and electron-plasmon [6] interactions and band-structure effects [7]. Its electronic properties are also very sensitive to stacking and the number of layers [8]. In bilayer graphene a gap that could be tuned by doping was observed [9]. For few-layer graphene, the parent compound, graphite, is the key to understanding these new phenomena. Interlayer coupling in an $A B$ stacking sequence leads to the formation of electron and hole pockets responsible for the semimetallic character in graphite. The linear dispersion is broken and only if we have an $A A$ stacking the linear dispersion remains. Nevertheless, at the $H$ point of graphite [2] the $\pi$ band dispersion is close to linear and has been interpreted as Dirac-fermion-like. Much less is known about the quantitative description of electron-electron correlations in these graphitic systems. Angle-resolved photoemission (ARPES) studies indicated that local density approximation (LDA) gives a dispersion that is too flat and a scaling has to be applied in order to fit the experimental dispersion of few-layer graphene and graphite. For the
PACS numbers: 79.60.-i, 71.10.- w, 71.20.Tx, 71.55.Ak

scaling, values of $\sim 10 \%[10,11]$ and $\sim 20 \%[12,13]$ have been reported in the literature. Correlation effects can cause the recently discovered quantitative shortcomings [14] of the double-resonant model [15] for Raman scattering in graphene and graphite. Furthermore, it is important to know the exact $k_{z}$ dispersion, because it is responsible for the conductivity perpendicular to the graphene layers.

In this Letter we report on a combined ARPES and theoretical $a b$ initio QP study of the three-dimensional $\pi$ band structure and the Fermi surface in graphite single crystals. ARPES is best for studying correlations since it probes the complex self-energy function which contains the electronic interactions. We elucidate the full electronic QP dispersion perpendicular to the layers and show directly the importance of the effects of electronic correlation and the influence on transport and resonance Raman in these layered materials. Experiments were done at BESSY II using the UE112-PGM2 beam line and a Scienta SES 100 analyzer yielding a total energy resolution of $15 \mathrm{meV}$ and a momentum resolution better than $0.01 \AA^{-1}$. Natural graphite single crystals with $A B$ stacking were cleaved in situ to give mirrorlike surfaces and were measured within $12 \mathrm{~h}$ after cleavage in a vacuum better than $10^{-10}$ mbar. The samples were mounted on a three axis manipulator that was cooled down by liquid He to $25 \mathrm{~K}$. The three-step model for ARPES is employed for data analysis [16]. After a dipole transition from the valence band to an unoccupied intermediate state, the electron travels to the sample surface where it is scattered into a free electron state outside the sample. When the electron leaves the sample, its wave vector component in the direc- 
tion perpendicular to the sample surface is not conserved which makes 3D band mapping a challenging task. We assume that the intermediate state dispersion is a parabola which is shifted downward in energy with respect to the vacuum level by $V_{0}$, the inner potential. We determine $V_{0}$ by finding the maximum splitting between the two valence $\pi$ bands, which occurs at $k_{z}=0$, i.e., $K$. This procedure yields $V_{0}=16.4 \pm 0.1 \mathrm{eV}$ and allows one to accurately determine the full $k_{z}$ dispersion by changing the photon energy [16]. Deviations from a parabolic dispersion of the intermediate state would change the $k_{z}$ assignment as described in [17-19]. However, as shown in detail below and in agreement with previous experiments $[2,12]$ for the electronic states of graphite close to the Fermi level this error is small.

The calculations of the electronic dispersion are performed on two levels. First, we calculate the Kohn-Sham band structure within the LDA to density-functional theory [20]. Wave functions are expanded in plane waves with an energy cutoff at $25 \mathrm{Ha}$. Core electrons are accounted for by Trouiller-Martins pseudopotentials. In the second step, we use the $G W$ approximation [21-23] to calculate the selfenergy corrections to the LDA dispersion [24]. For the calculation of the dielectric function $\epsilon(\omega, q)$ we use a $15 \times$ $15 \times 5$ Monkhorst-Pack $k$ sampling of the first Brillouin zone (BZ), and conduction band states with energies up to $100 \mathrm{eV}$ above the valence band ( 80 bands). The calculations are converged to $10 \mathrm{meV}$. Since the $G W$ calculations are computationally expensive a tight-binding (TB) fit to $G W$ (TB- $G W$ ) was performed in order to rapidly calculate the QP bands.

We now turn to the comparison of the ARPES data to the calculated band structures. In Fig. 1 we show raw electron dispersion curve (EDC) data and cuts of the $\pi$ bands that intersect the corners of the $\mathrm{BZ}$ at $k_{z}=0.46 \AA^{-1}$ (very close to the $H$ point), at $k_{z}=0.22 \AA^{-1}$ and at $k_{z}=0$ (the $K$ point). The cuts are done along the $k_{y}$ axis [see the coordinate system in Fig. 2(a)]. It is clear that for the cut through the $H$ point, the bands are almost linear and only one $\pi$ valence band can be seen, whereas at the $K$ point, the degeneracy is lifted and the bands are parabolic. The energy separation between the two valence $\pi$ bands is getting smaller when moving away from $K$ in the $k_{z}$ direction. The LDA and $G W$ calculation data along the measured cut are also shown in Fig. 1. LDA underestimates the slope of the bands while the self-energy corrections from the $G W$ calculation yield a better agreement. In the inset of Fig. 1(b) we show the gap $\Delta$ at $H$ obtained by $G W$. It is clear that the dispersion is not linear close to $H$ and the concept of Dirac fermions breaks down. This is important for transport properties; however, for an estimation of the band maxima a linear extrapolation above $E_{F}$ is a good approximation due to the extremely high curvature at the maximum of the holelike band at $H$.

We can obtain the Fermi surface of the holelike band with a band maximum above $E_{F}$ by the following method: we fit a linear dispersion to the EDC maxima in a region below $E_{F}$ and then extrapolate it to find the crossing of the extrapolated band with the Fermi level. A dispersion very close to linear is predicted by calculation [inset of Fig. 1(b)] as the functional form of the band at $H$. From the crossing of the linear dispersion with $E_{F}$, we can then obtain an estimate for the cross section of the hole pocket at $H$. Such a procedure is needed in order to determine the maximum of the sparsely populated band and has been used in the literature [2]. For points with $k_{z}<0.5 c$, we choose a quadratic fit as the functional form around the band maximum $(c$ is the length of the reciprocal lattice vector in $k_{z}$ direction). Assuming electron-hole symmetry for the sparsely occupied band, we can estimate the cross
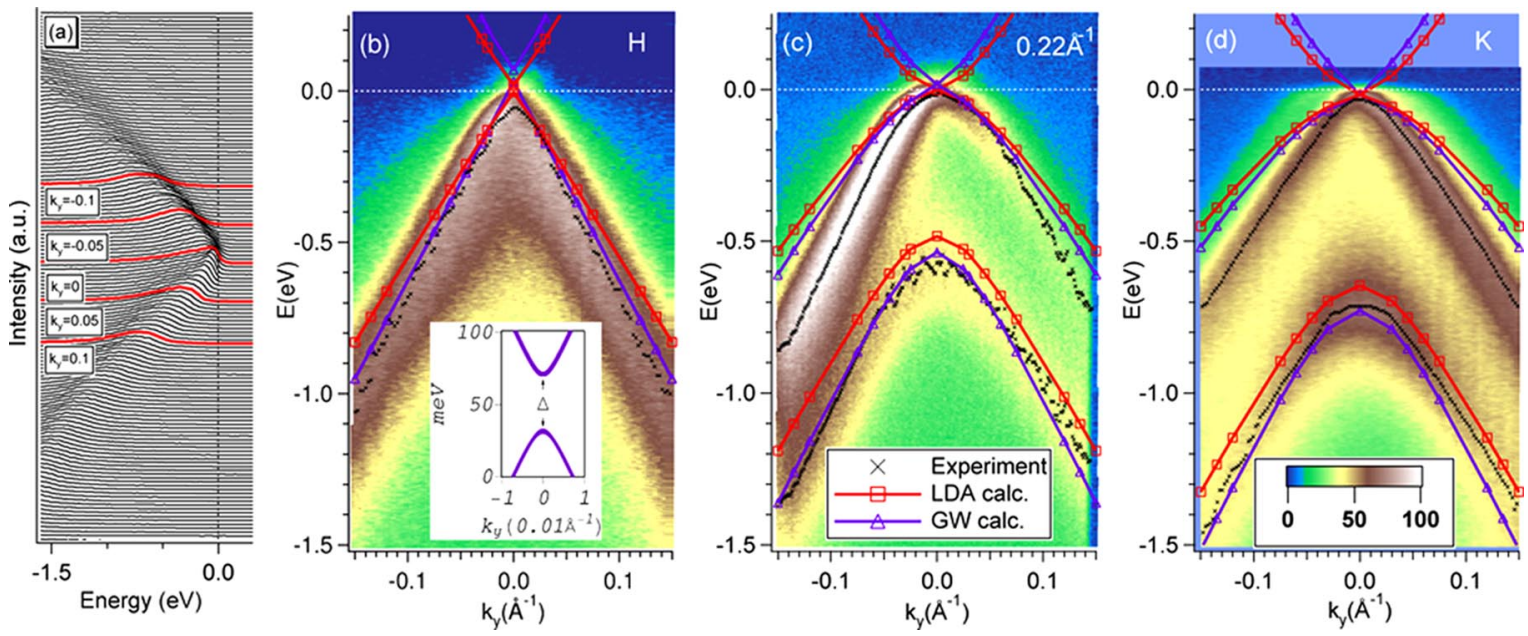

FIG. 1 (color online). (a) Raw EDC data for the cut through $H$. Cuts at special $k_{y}$ (values in $\AA^{-1}$ ) are indicated by thick lines. Photoemission intensity of cuts through the 3D BZ of graphite at (b) $H$, (c) $k_{z}=0.22 \AA^{-1}$, and (d) $K$ point. Along with the photoemission intensity, we show results of LDA $(\square)$ and $G W(\triangle)$ calculations. The inset in (b) shows a magnification of the TB-GW calculation for the small hole pocket and the gap $\Delta$. The photon energies used were (b) $100 \mathrm{eV}$, (c) $25 \mathrm{eV}$, and (d) $83 \mathrm{eV}$. The intensity in (c) is multiplied by a factor of 2 in order to visualize the low intensity lower $\pi$ band. 


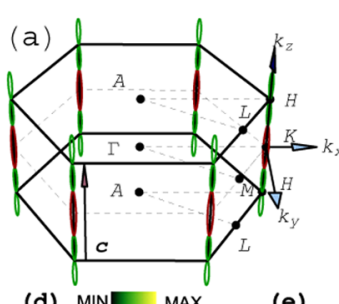

(b) $0.05 \AA^{-1}$

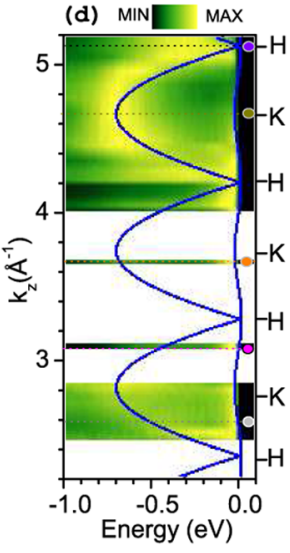

(e)
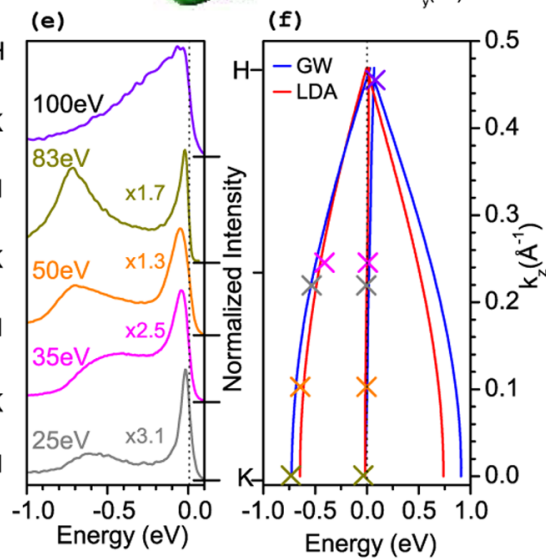

FIG. 2 (color online). (a) Graphite BZ with the high-symmetry points, reciprocal lattice vector $\mathbf{c}$, and the coordinate system. The curves around $K(H)$ are the envelopes of the measured electron (hole) pockets. (b) depicts the interpolation of the 3D Fermi surface to experimental data. (c) Obtaining band maxima: crosses denote symmetrized EDC data and the line an extrapolation. The hole pocket cross section with $E_{F}$ is denoted by circles. The arrow denotes the band maximum at $H$. (d) photoemission intensity along the $K H$ direction for photon energies from 22 to $105 \mathrm{eV}$ and $G W$ calculations (solid line). The five colored points along $K H$ denote $k_{z}$ for which we evaluate the EDC maxima. (e) Raw EDCs for the $k_{z}$ denoted by colored points. (f) The experimental EDC maxima and the calculated LDA and $G W$ dispersion.

sections with $E_{F}$. From these data and some higher energy points the Fermi surface could be interpolated with the standard tight-binding Hamiltonian [25].

The pockets we obtained are shown in Fig. 2(a) along with a sketch of the BZ and the coordinate system and reciprocal lattice vector $\mathbf{c}$. A detailed magnification of the tiny electron and hole pockets is shown in Fig. 2(b) with the aspect ratio enlarged by a factor of 30 for easier viewing. The surfaces around $K$ and $H$ points are the electron and hole pockets, respectively. The volumes inside the Fermi surface based on experiment yield carrier densities of $n_{e}=2.3 \times 10^{19} \mathrm{~cm}^{-3}$ electrons and $n_{h}=1.8 \times$ $10^{19} \mathrm{~cm}^{-3}$ holes. In Fig. 2(c) we illustrate the procedure to obtain the $\pi$ band maxima at $H$ which is located above $E_{F}$. In Fig. 2(d) we show the photoemission intensity along the $K H$ direction for photon energies between 22 and $105 \mathrm{eV}$. In Fig. 2(e) the raw EDC data for selected cuts along $K H$ are depicted and in Fig. 2(f) the EDC band maxima (folded back to the first BZ) are compared to theory. The experimental results and theory have the same trend, which is that the lower $\pi$ valence band comes close to the upper one when moving from the $K$ to the $H$
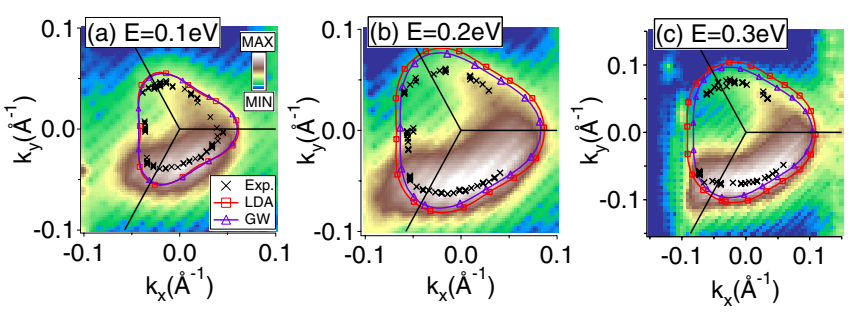

FIG. 3 (color online). Equienergy contours of the photoemission intensity (logarithmic scale) around the $K H$ axis for $k_{z}=$ $0.22 \AA^{-1}$ corresponding to Fig. 1(c). Experimental EDC maxima are denoted by crosses. Lines depict TB- $G W(\triangle)$ and LDA ( $\square$ ) calculations, respectively. Trigonal warping and the strong asymmetry in the intensity can clearly be seen.

point. The experimental splitting between the $\pi$ valence bands is generally better described by the $G W$ approximation than by LDA.

In Fig. 3 the trigonal warping effect is investigated. In order to accurately compare to calculations we evaluate the maxima of the EDCs for equienergy contours in the $k_{x}, k_{y}$ plane. We have chosen $k_{z}=0.22 \AA^{-1}$, which lies approximately halfway between $K$ and $H$. The experimental and calculated $G W$ and LDA contours for three energies 0.1$0.3 \mathrm{eV}$ are shown in (a)-(c). LDA not only underestimates the slope of the bands, but also a simple scaling does not work because a different scaling would be needed for $\Gamma$ and $M$ directions. Although the $G W$ approximation does not describe the experimental data in all directions, it is always closer to the experiment than the LDA. Interestingly, it is in perfect agreement with the experiment at the $H$ point while the agreement is worse when going away from $H$. This discrepancy might be due to the $G W$ approximation and resolved by inclusion of higher orders in the calculation of the self-energy correction in a future work.

A strong asymmetry around the $K H$ axis in energy (trigonal warping effect) and photoemission intensity is observed. The trigonal warping effect of the ARPES data is underestimated by the LDA and again better described in the $G W$ approximation. Compared to graphene we find that trigonal warping is higher by a factor of $\sim 1.5$ when considering the distances in the two high-symmetry directions for an equienergy contour of $0.1 \mathrm{eV}$ in Fig. 3. The strong asymmetry in the photoemission intensity seen in this figure is attributed to the dipole matrix element for the transition from a valence band state to an unoccupied state $25 \mathrm{eV}$ above $E_{F}$ [26].

We now turn to a quantitative assessment of electron correlation effects and their implications for Raman and transport studies of graphite and related materials. The experimental and calculated values for the energy separation of valence bands at $K$, the gap $\Delta$ at $H$, and the Fermi velocity $v_{F}$ are listed in Table I. We obtain the Fermi velocities of the linear band in the $H A$ and $H L$ directions in a region up to $1.5 \mathrm{eV}$ below $E_{F}$. Since the trigonal warping effect has a minimum in this $k_{z}$ plane, the differences in these two directions are small. The LDA values for 
TABLE I. Fermi velocities at $H$ (in $10^{6} \mathrm{~m} / \mathrm{s}$ ), valence band energy separation at $K$, and $\Delta$ obtained by experiment and from calculations.

\begin{tabular}{lcccc}
\hline \hline Method & $\begin{array}{c}v_{F} \\
(H A)\end{array}$ & $\begin{array}{c}\boldsymbol{v}_{F} \\
(H L)\end{array}$ & $\begin{array}{c}\text { Energy separation } \\
(\mathrm{eV})\end{array}$ & $\begin{array}{c}\Delta \\
(\mathrm{eV})\end{array}$ \\
\hline ARPES & $1.06 \pm 0.1$ & $1.05 \pm 0.1$ & $0.71 \pm 0.015$ & - \\
$G W$ & 0.99 & 1.02 & 0.71 & 0.037 \\
LDA & 0.84 & 0.87 & 0.62 & 0.022 \\
\hline \hline
\end{tabular}

$v_{F}$ are far off, but the $G W$ is in excellent agreement with the experiment.

The dispersion of $D$ and $G^{\prime}$ double-resonant Raman (DRR) bands [15] with laser energy is proportional to $v_{\mathrm{Ph}} / v_{F}$, where $v_{\mathrm{Ph}}$ is the slope of the transversal optical phonon branch going through $K(H)$. The electronic dispersion in $k_{z}$ broadens the DRR dispersion and with the present data DRR spectra for 3D graphite can be calculated taking into account the full electronic dispersion. For a given phonon dispersion we thus expect the present data to reduce the slope of the calculated DRR bands since the $v_{F}$ from our experiment are blueshifted with respect to the LDA calculation.

In addition, the gap $\Delta$ shows a large difference in values with a $G W$ value that is 5 times the TB parameter that was originally fit to a magnetoreflectance experiment [25]. Such a deviation can be attributed to stacking faults such as $A A$ stacking, which has $\Delta=0$. For a sample consisting of $A B$ stacking with many stacking faults the effectively measured $\Delta$ would be lower explaining the experimental value of $\Delta=0.008 \mathrm{eV}$ in pyrolytic graphite [25]. A finite value of $\Delta$ causes a breakdown of the linear bands at the $H$ point. Their shape becomes parabolic with a very large curvature and thus a small absolute value of the effective mass.

From the 3D Fermi surface the transport properties of graphite can be calculated from a Drude model where the carrier densities and the effective masses can be directly taken from the ARPES experiment. For the $k_{z}$ averaged electron and hole masses, we obtain $m_{e}^{*}=0.10 m_{0}$ and $m_{h}^{*}=-0.04 m_{0}$, respectively, that is in good agreement with a previous magnetoreflectance study $\left(m_{0}\right.$ is the free electron mass) [27]. For the scattering time $\tau$ we use a value of $\tau \sim 200$ fs from pump-probe experiments [28]. We get a dc conductivity $\sigma=n_{e} e^{2} \tau / m_{e}^{*}+n_{h} e^{2} \tau / m_{h}^{*}$ of $\sigma=3.9 \times 10^{4} \Omega^{-1} \mathrm{~cm}^{-1}$. The values agree nicely with transport measurements that yield $2.5 \times 10^{5} \Omega^{-1} \mathrm{~cm}^{-1}$ [29] and $2.5 \times 10^{4} \Omega^{-1} \mathrm{~cm}^{-1}$ [25] when considering that the literature values differ by an order of magnitude.

In conclusion we have performed ARPES of graphite and compared the measured QP dispersion to $a b$ initio calculations. We have found that the band dispersions are better described by the $G W$ approximation; however, around the $K$ point that agreement becomes poorer and many-body approximation schemes beyond $G W$ may improve this result. Moreover, for this system the concept of Dirac fermions breaks down. This highlights the impor- tance of electron correlations which renormalize the electronic dispersion resulting in a blueshift of the gap at $H$ and an increased $v_{F}$. A direct comparison of a scaled LDA QP dispersion to transport and Raman experiments is not possible due to an anisotropy of the scaling needed for the LDA around the $K H$ axis. TB- $G W$ allowed us rapid calculation of the QP bands.

A. G. acknowledges a Marie Curie Individual Fellowship (COMTRANS) from the European Union. T.P. acknowledges DFG Projects No. PI 440/3 and No. 440/4. C. A. and L. W. acknowledge support from the French national research agency. A. R. is supported by the EC Network of Excellence Nanoquanta (No. NMP4-CT2004-500198), SANES project (No. NMP4-CT-2006017310), Basque Country University (SGIker Arina), and MEC (No. FIS2007-65702-C02-01). Calculations were performed at Barcelona supercomputing center and at Idris (Paris). We thank Andrea Marini for making his $G W$-code SELF available to us.

[1] A. Geim and K. Novoselov, Nat. Mater. 6, 183 (2007).

[2] S. Y. Zhou et al., Nature Phys. 69, 245419 (2006).

[3] M. I. Katsnelson, K. S. Novoselov, and A. K. Geim, Nature Phys. 2, 620 (2006).

[4] K. Novoselov et al., Science 306, 666 (2004).

[5] S. Y. Zhou, G. H. Gweon, and A. Lanzara, Ann. Phys. (N.Y.) 321, 1730 (2006).

[6] A. Bostwick et al., Nature Phys. 3, 36 (2007).

[7] C. Spataru et al., Phys. Rev. Lett. 87, 246405 (2001).

[8] S. Latil and L. Henrard, Phys. Rev. Lett. 97, 036803 (2006).

[9] T. Ohta et al., Science 313, 951 (2006).

[10] C. Heske et al., Phys. Rev. B 59, 4680 (1999).

[11] V. Strocov et al., Phys. Rev. B 64, 075105 (2001).

[12] T. Ohta et al., Phys. Rev. Lett. 98, 206802 (2007).

[13] S. Y. Zhou et al., Phys. Rev. B 71, 161403 (2005).

[14] D. Graf et al., Nano Lett. 7, 238 (2007).

[15] C. Thomsen and S. Reich, Phys. Rev. Lett. 85, 5214 (2000).

[16] S. Hüfner, Photoelectron Spectroscopy (Springer-Verlag, Berlin, 1996).

[17] V. Strocov et al., Phys. Rev. B 61, 4994 (2000).

[18] N. Barrett et al., Phys. Rev. B 71, 035427 (2005).

[19] E. Krasovskii et al., Phys. Rev. Lett. 98, 217604 (2007).

[20] X. Gonze et al., Comput. Mater. Sci., 25, 478 (2002).

[21] M. S. Hybertsen and S. G. Louie, Phys. Rev. B 34, 5390 (1986).

[22] L. Hedin, Phys. Rev. 139, A796 (1965).

[23] S. G. Louie, Topics in Computational Materials Science, edited by C. Y. Fong (World Scientific, Singapore, 1997), p. 96.

[24] By A. Marini, http://www.fisica.uniroma2.it/ self/.

[25] M.S. Dresselhaus and G. Dresselhaus, Adv. Phys. 30, 139 (1981).

[26] E. L. Shirley et al., Phys. Rev. B 51, 13614 (1995).

[27] E. Mendez, T. Chieu, and N. K. M. Dresselhaus, Solid State Commun. 33, 837 (1980).

[28] G. Moos et al., Phys. Rev. Lett. 87, 267402 (2001).

[29] D. Morelli and C. Uher, Phys. Rev. B 30, 1080 (1984). 